BMJ Open Sport \& Exercise Medicine

\title{
Mental health symptoms in electronic football players
}

Ana Monteiro Pereira (D) , ${ }^{1,2}$ Pedro Teques, ${ }^{1,3,4}$ Evert Verhagen (i) ,
Vincent Gouttebarge (1) ,6,7,8 Pedro Figueiredo, ${ }^{1,9,10}$ João Brito $^{1}$

To cite: Pereira AM, Teques $P$, Verhagen $\mathrm{E}$, et al. Mental health symptoms in electronic football players. BMJ Open Sport \& Exercise Medicine 2021;7:e001149. doi:10.1136/ bmjsem-2021-001149

Accepted 27 September 2021

Check for updates

(c) Author(s) (or their employer(s)) 2021. Re-use permitted under CC BY-NC. No commercial re-use. See rights and permissions. Published by BMJ.

For numbered affiliations see end of article.

Correspondence to Ana Monteiro Pereira; ana.pereira@fpf.pt

\section{ABSTRACT}

Background The escalated competitive pressure and professionalisation of esports players could predispose them to mental health symptoms. We aimed to develop a model to explore the association between distress and anxiety/depression symptoms and potential associations between adaptive or maladaptive coping strategies, sleeping disturbance, alcohol consumption and eating habits in electronic football players.

Methods The present exploratory cross-sectional study includes the Distress Screener, General Health Questionnaire, Brief Cope, Sleep Disturbance Domain of the Patient-Reported Outcomes Measurement Information System, Alcohol Use Disorders Identification Test Consumption and a set of questions regarding eating habits. A two-step robust maximum likelihood method of the Structural Equation Modelling approach was used. Results Both measurement model $\left(\chi^{2} / \mathrm{df}=1065.04\right.$ (637), $\mathrm{p}<0.001$; Tucker-Lewis Index $(\mathrm{TLI})=0.91$, Comparative Fit Index $(\mathrm{CFI})=0.92$, standardised root mean residual $(\mathrm{SRMR})=0.07$ and root mean square error of approximation (RMSEA) $=0.05 ; 95 \% \mathrm{Cl} 0.043$ to 0.053 ) and hypothesised structural model $\left(\chi^{2} / \mathrm{df}=1131.98(648) ; p<0.001\right.$; $\mathrm{TLI}=0.90, \mathrm{CFI}=0.91, \mathrm{SRMR}=0.07$ and $\mathrm{RMSEA}=0.05$; $95 \% \mathrm{Cl} 0.054$ to 0.06 ) showed an adequate fit to the data. Stress was positively related with depression and anxiety symptoms. Only anxiety symptoms were linked with coping strategies. Maladaptive coping strategies were related to sleep disturbance, alcohol consumption and poor eating habits, whereas adaptive coping strategies were associated with less sleep disturbance, reduced alcohol consumption and better eating habits.

Conclusion An integrative approach to help electronic football players when early signs of distress are present might help avoid other mental health symptoms that interfere with players' well-being and overall health. Additionally, adequate coping strategies to manage anxiety symptoms due to distress may help counteract potential negative consequences for lifestyle habits in electronic football players.

\section{INTRODUCTION}

Esports are a worldwide phenomenon, with more than 2.7 billion video gamers ${ }^{1}$ and an estimated global audience of 474 million people worldwide. ${ }^{2}$ In esports, gamers compete in organised and regulated competitions of different games in a virtual, 'electronic' environment with a specific

\section{Key messages}

What is already known

- There is increased pressure in the esports scene which is acerbated by the increased social and media impact of esports.

- It has already been proposed that several factors could predispose esports players to experience several health-related problems, including mental health symptoms, that might affect their performance.

- The scientific literature is still scarce on mental health consequences of esports practice.

What are the new findings

- The prevalence of mental health symptoms electronic football players is high, with $22 \%$ showing anxiety symptoms and $37 \%$ showing depression symptoms.

- There were $45 \%$ electronic football players with sleep disturbance, $25 \%$ with alcohol consumption and $26 \%$ with poor eating habits.

- Distress was positively associated with depression and anxiety symptoms, but only anxiety symptoms were linked with active coping strategies.

- Adaptative coping strategies were negatively related to sleep disturbance, alcohol consumption and poor eating habits, while maladaptive coping strategies were positively related to sleep disturbance, alcohol consumption and poor eating habits.

goal/prize. ${ }^{2}{ }^{3}$ However, rather than a recreational activity, esports players now aim for sponsorship, professionalisation and bigger competition rewards. That increases the pressure for better performances, which is acerbated by the increased social and media impact of esports. ${ }^{3}$

Like traditional athletes, ${ }^{4}$ esports players need training and expertise of mental and physical abilities to succeed. ${ }^{5-7}$ With the stakes rising in the esports scene, it has already been proposed that several factors could predispose esports players to experience several health-related problems, including mental health symptoms, ${ }^{389}$ that can hinder performance and well-being. ${ }^{10}$ To note, mental health problems are major causes of morbidity and disability worldwide, with 322 million people being estimated to 


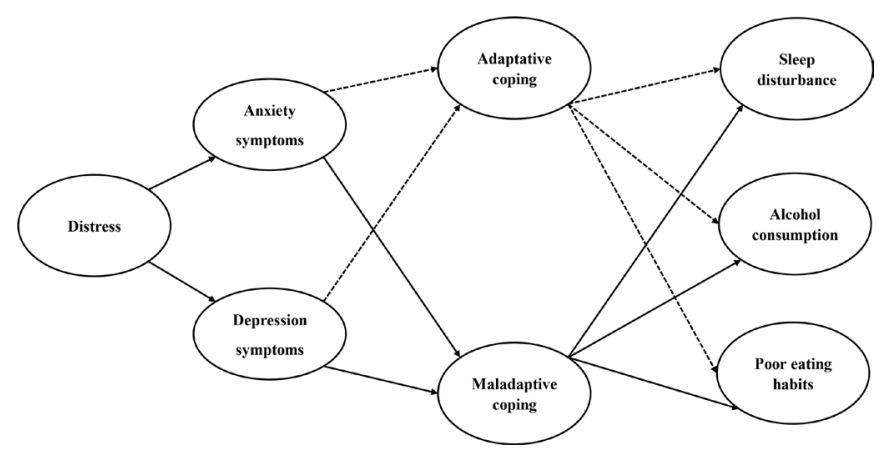

Figure 1 Hypothesised path model for the relationships between distress in eFootball players with anxiety/ depression symptoms, coping strategies and the outcome variables sleep disturbance, alcohol consumption and poor eating habits. Note: positive paths in continuous lines and negative paths in dashed lines.

suffer from depression and 264 million from anxiety. ${ }^{11}$ Stress, alcohol consumption, sleep problems and dietary risks are also risk factors that are also account for on the Global Burden of Diseases, ${ }^{12}$ and might predispose to other noncommunicable diseases (eg, digestive diseases, cardiovascular diseases, diabetes, obesity or even cancer). ${ }^{13} 14$ According to the International Olympic Committee consensus statement on mental health in elite athletes, ${ }^{15}$ 'mental health symptoms are more common, may be significant but do not occur in a pattern meeting specific diagnostic criteria and do not necessarily cause significant distress or functional impairment'. However, even with millions of people involved directly or indirectly with esports practice, the scientific literature is still scarce on this topic.

Therefore, it will be important to understand the prevalence of mental health symptoms and coping strategies in esports players and their association. Preliminary evidence suggests that esports players may be at a risk of mental health problems relative to their traditional athletes' counterparts. For such, the present study aimed to develop a model to explore the association between distress and anxiety/depression symptoms, as well as sleep disturbance, adverse alcohol consumption and poor eating habits based on adaptive or maladaptive symptoms in electronic football (eFootball) players, an esports modality related to sports. We hypothesised (figure 1) that in eFootball players, distress symptoms are positively related with depression (hypothesis 1 ) and anxiety symptoms (hypothesis 2), and that both depression (hypothesis 3) and anxiety symptoms (hypothesis 4) are positively connected to maladaptive coping strategies and negatively related to adaptative coping strategies. Also, we hypothesised that maladaptive mechanisms positively associate with the outcome variables sleep disturbance, alcohol consumption and poor eating habits (hypothesis 5). In contrast, adaptative mechanisms have a negative association with the outcome variables (hypothesis 6).

\section{METHODS}

\section{Design}

The current Structural Equation Modelling (SEM) study employed a cross-sectional design based on a structured online survey comprising the Distress Screener, ${ }^{16}$ the 12-item General Health Questionnaire (GHQ-12), ${ }^{17}$ Brief Cope, ${ }^{18}$ the Sleep Disturbance Domain of the Patient-Reported Outcomes Measurement Information System (PROMIS-SD), ${ }^{19}$ Alcohol Use Disorders Identification Test Consumption (AUDIT-C) ${ }^{20}$ and a set of questions regarding eating habits. ${ }^{21}$ The measuring tools chosen were based on a previous investigation regarding the prevalence of common mental health symptoms in professional football players. ${ }^{22}$ The Portuguese versions of GHQ-12, Brief Cope and AUDIT-C were employed, while previously translated versions (Teques P, Araújo D. Indicadores de Saúde Mental de Futebolistas Profissionais. 2019. XX Jornadas da Sociedade Portuguesa de Psicologia do Desporto) from the Distress Screener, PROMIS-SD and a set of questions regarding eating habits were employed. A questionnaire to perform a sociodemographic characterisation, including questions related to esports practice and competitive status, was included; the procedures have been validated by the Portuguese Football Association (FPF) Data Protection Office and the FPF Esports department.

\section{Study setting and participants}

The study involved a convenience sample of eFootball players registered on the FPF Esports online platform. Registration on FPF Esports was a requirement to participate in the different esports tournaments and competitive events organised by the FPF Esports department. For that, only information regarding name, date of birth and contact e-mail was required, without the need to participate in esports competitions or to keep up a determined FIFA rank.

All 9099 eFootball players registered on FPF Esports in December 2019 received via e-mail an online survey, written in Portuguese, using SurveyMonkey. The time to complete the full questionnaire was estimated to be 12-15 min. Participants younger than 18 years were excluded from the analysis.

\section{Symptoms related to mental health problems and coping Distress}

The Distress Screener consists of three items (eg, 'During the past week, did you suffer from worry?') scored between 1 , if 'never', to 3 , if 'regularly or very frequently'. The score is obtained by summing up the answers for the three items. The presence of distress is assumed when a score of 4 or above is obtained. ${ }^{23}$

\section{Depression and anxiety symptoms}

Evaluation of symptoms related to anxiety (eg, 'Have you recently lost much sleep over worry?') and depression (eg, 'Have you recently been feeling unhappy and depressed?') in the previous 4 weeks was performed 
using the anxiety/insomnia and severe depression factors of the Portuguese version of GHQ-12. ${ }^{24}$ A Likert 4-point scoring method was used to calculate the total score, where the higher value represented more severe condition. ${ }^{25}$ Following Goldberg and colleagues, ${ }^{17}$ scores higher than 2 were considered to establish the presence of anxiety/depression.

\section{Adaptative and maladaptive coping}

To assess situational coping strategies in eFootball players, the Portuguese version of Brief Cope ${ }^{26}$ was employed. The Brief Cope is a self-report questionnaire with 28 items (eg, 'I've been refusing to believe that it has happened') answered on a 4-point Likert-type scale (ranging from $1=$ 'I have not used this at all' to $4=$ ='I have used it a lot'). According to Carver, ${ }^{18}$ we used the two broad dimensions that cover the 14 scales of Brief Cope: (1) adaptive coping strategies described as 'active coping, acceptance, humour, religion, planning, positive reframing, and using instrumental and emotional support', and (2) maladaptive coping strategies, such as 'behavioural disengagement, denial, self-blame, self-distraction, substance use, and venting negative emotion'.

\section{Sleep disturbance}

Patient-Reported Outcomes Measurement Information System comprises different validated person-centred measures to evaluate and monitor physical, mental and social health in adults and children. Those measures, including validated translated versions, can be found online (wwwhealthmeasuresnet). In the present study, PROMIS-SD $^{19}$ was applied to assess difficulties and concerns with getting to sleep or staying asleep, and perceptions of the adequacy and satisfaction with sleep ${ }^{19} 27$ in the past 7 days. All four items (eg, 'My sleep was restless') were responded to on a 5-point Likert Scale with one for 'not at all' and 5 for 'very much'. Total score, ranging from 1 to 20 , results from summing all four items. We considered a t-score higher than 50 as indicative of sleep disturbance.

\section{Alcohol consumption}

The three-item version of the AUDIT (known as AUDIT-C) was used to screen for alcohol misuse. ${ }^{20}$ AUDIT-C evaluates frequency and quantity of alcohol consumption and frequency of binge drinking, considering the total score the one obtained by summing up the three items, ranging from $0-12$. Scores of 5 or more in men and 4 or more in women indicate a high probability of excessive alcohol misuse. Given the small proportion of women, the probability of excessive alcohol misuse was considered when a score of 5 or more was obtained.

\section{Eating habits}

Eating habits were evaluated using the questionnaire developed by Van der Veer et al. ${ }^{21}$ In their study, eating habits were assessed by asking how many days per week the participant 'eat healthy', 'eat regularly throughout the day', 'eat the meal before 10:00 AM' and 'eat the last meal before 8:30 PM'. We considered poor habits if the respondent regularly ate throughout the day, having breakfast before 10:30 and having their last meal before 20:30, less than 3 days/week.

\section{Statistical analysis}

Descriptive data analyses for quantitative variables (eg, age) were described by the mean (M) and SD. In contrast, categorical variables (eg, preferred game and type of esports practice) were described using absolute (n) and relative $(\%)$ frequencies. The prevalence of variables under study was calculated using Wilson Score interval and $95 \% \mathrm{CI}$, and a t-score of $>50$ was used for maladaptive/adaptative coping and a t-score $>50$ for sleep disturbance. GPower V.3. $1^{28}$ was used to perform a priori power analysis. The required sample size was estimated to be 215 participants, based on the following input parameters: effect size $\mathrm{f} 2=0.1, \alpha=0.05$ and statistical power $=0.95$; and five predictors (ie, distress, anxiety symptoms, depression symptoms, adaptive and maladaptive coping). Non-descriptive data analysis was performed applying a two-step robust maximum likelihood method of SEM approach with IBM AMOS V.23 (IBM Corp., Armonk, New York, USA).$^{29}$ For such, the variables' quality adjustment to its indicators was analysed with confirmatory factor analysis (CFA). To test the significance of the variables and direct and indirect effects, bootstrap resampling procedure (1000 bootstrap samples) with 95\% bias-corrected CI was used. An indirect effect was considered significant $(\alpha=0.05)$ if the $95 \%$ CI did not include zero. ${ }^{30}$ CFA was used to evaluate the quality of the variable's adjustment in the measurement model. Four indexes were considered to estimate the local adjustment of the model to the data ${ }^{29}$ : Comparative Fit Index (CFI) and Tucker-Lewis Index (TLI) of $>0.90$, root mean square error of approximation (RMSEA) and standardised root mean residual (SRMR) of $<0.08$. Additionally, to assess the model's global adjustment, composite reliability (CR) of $\geq 0.70$ was used to evaluate the reliability and internal consistency, and variance average extracted (AVE) value of $\geq 0.50$ was used to evaluate each construct convergent validity. The discriminant validity will be assumed when AVE values are greater than the squared correlations between other constructs. ${ }^{29}$ Additionally, to verify collinearity within all study variables, variance inflation factors of $<10$ were accepted to conduct regression analysis. ${ }^{29}$

\section{RESULTS}

\section{Participants}

The online survey was fully completed by 292 eFootball players (response rate of $3.2 \%$ ). The mean age was 27.0 48.1 years (ranging from 18 to 72 years old), and $97.6 \%$ were men. Interestingly, $62.7 \%$ of the participants had been previously registered in a national sports federation, and $11 \%$ were still registered in a sports modality besides practising esports (table 1 ).

Sample characterisation regarding esports practice is shown in table 2 . The eFootball players mostly played 1 
Table 1 Sample characteristics

\begin{tabular}{lc}
\hline Variables & $\mathbf{n}(\%)$ \\
\hline Sex & \\
$\quad$ Male & $285(97.6)$ \\
$\quad$ Female & $7(2.4)$ \\
Nationality & \\
$\quad$ Portuguese & $280(95.9)$ \\
\hline Other nationality & $12(4.1)$ \\
\hline Education & \\
Did not study/finish any level & $3(1.0)$ \\
Elementary school & $9(3.1)$ \\
\hline Non-academic course & $64(21.9)$ \\
\hline High school & $144(49.3)$ \\
\hline University & $72(24.7)$ \\
Sports federation status & \\
$\quad$ Non-registered & $77(26.4)$ \\
\hline Previously registered & $183(62.7)$ \\
\hline Still registered & $32(11.0)$ \\
\hline
\end{tabular}

\begin{tabular}{lc|}
\hline \multicolumn{2}{|l|}{ Table 2 Characterisation of esports practice } \\
\hline Variables & $\mathbf{n}(\%)$ \\
\hline EA FIFA game mode* & $181(62)$ \\
\hline 1 vs 1 & $35(12)$ \\
\hline 2 vs 2 & $182(62.3)$ \\
\hline Pro Clubs & \\
\hline Preferred game platform & $247(84.6)$ \\
\hline PS4 & $15(5.1)$ \\
\hline Xbox & $29(9.9)$ \\
\hline Personal computer & $1(0.3)$ \\
\hline Other & $18(6.2)$ \\
\hline Type of esports practice $\dagger$ & $274(93.8)$ \\
\hline Professional & \\
\hline Recreational & $178(61.0)$ \\
\hline Level of esports competitions & $11(3.8)$ \\
\hline National & $103(35.3)$ \\
\hline International & $158(54.1)$ \\
\hline Both & $134(45.9)$ \\
\hline Included in an esports team & $233(79.8)$ \\
\hline Yes & $59(20.2)$ \\
\hline No & \\
\hline Practice of other video games & \\
\hline Yes & \\
\hline No & \\
\hline
\end{tabular}

*More than one response was accepted.

†Professional practice='My eFootball practice is remunerated'; recreational practice='My eFootball practice is not remunerated'. versus 1 and in Pro Clubs mode, with 247 players using PS4 console. Even with only $6.2 \% \quad(n=18)$ being professional eFootball players (ie, they have eFootball as a full-time paid activity), 39.1\% ( $\mathrm{n}=114)$ competed in international events and $54.1 \%(\mathrm{n}=158)$ were included in an esports team.

\section{Preliminary analysis}

Before applying a two-step robust maximum likelihood method of the SEM approach, a preliminary analysis was performed. Mardia's coefficient (171.55) exceeded the expected values for the multivariate normality, leading to a Bollen-Stine bootstrap for subsequent analysis. ${ }^{31}$ Additionally, variance inflation factors ranged from 1.05 (depression) to 2.24 (anxiety), showing acceptable conditions to conduct regression analysis. ${ }^{29}$

\section{Measurement model}

Mental health symptoms and coping strategies were prevalent in eFootball players. Almost a quarter of the participants demonstrated symptoms of distress and depression, and almost half of them presented sleep disturbance (table 3 ).

The model showed convergent validity for distress, anxiety symptoms, and depression symptoms and alcohol consumption (AVE >0.50). In addition, all variables showed discriminant validity since the AVE of each latent variable was higher than the square of the correlations between all variables. Reliability was demonstrated for all variables $(\mathrm{CR}>70)$, except for sleep disturbance and eating habits $(\mathrm{CR}<0.70)$. Still, since those variables were theoretically important to the model, they were maintained. Means, SD, prevalence, squared correlations, reliability, convergent and discriminant validity among all scales used are shown in table 3 .

Regarding the correlation between variables, most of the variables were related to each other. Distress was related to all variables, showing a positive association with depression symptoms, anxiety symptoms, maladaptive coping strategies, and the outcome variables sleep disturbance, alcohol consumption and eating habits, and a negative association with adaptative coping strategies. Depression symptoms were associated only with anxiety symptoms, with no significant association for the other variables related to mental health symptoms. Conversely, anxiety symptoms were related to adaptive and maladaptive coping strategies, with significant positive associations with outcome variables. The two dimensions of coping strategies were positively associated. Adaptive coping was negatively related to sleep disturbance, alcohol consumption and poor eating habits. Conversely, maladaptive strategies were positively associated with sleep disturbance but not with the other outcome variables. Similarly, no association between sleep disturbance and alcohol consumption was detected.

The assessment of the measurement model included distress, anxiety symptoms, depression symptoms, coping strategies, sleep disturbance, alcohol consumption and 
Table 3 Means, SD, squared correlations, reliability, convergent and discriminant validity among all scales

\begin{tabular}{|c|c|c|c|c|c|c|c|c|}
\hline & 1 & 2 & 3 & 4 & 5 & 6 & 7 & 8 \\
\hline 1. Distress & 1.00 & & & & & & & \\
\hline 2. Depression & $0.03^{\star *}$ & 1.00 & & & & & & \\
\hline 3. Anxiety & $0.57^{\star *}$ & $0.06^{\star \star}$ & 1.00 & & & & & \\
\hline 4. Maladaptive & $0.23^{\star *}$ & 0.01 & $0.14^{\star *}$ & 1.00 & & & & \\
\hline 5. Adaptive & $-0.01^{*}$ & -0.01 & $-0.06^{\star \star}$ & $0.17^{\star \star}$ & 1.00 & & & \\
\hline 6. Sleep disturbance & $0.10^{\star *}$ & 0.01 & $0.14^{\star *}$ & $0.03^{*}$ & $-0.05^{\star *}$ & 1.00 & & \\
\hline 7. Alcohol consumption & $0.02^{*}$ & 0.01 & $0.05^{\star *}$ & 0.01 & $-0.03^{\star *}$ & 0.01 & 1.00 & \\
\hline 8. Eating habits & $0.05^{*}$ & 0.01 & $0.04^{\star *}$ & 0.01 & $-0.02^{*}$ & $0.04^{\star *}$ & $0.14^{\star \star}$ & 1.00 \\
\hline$\%(95 \% \mathrm{Cl}) / \mathrm{t}$-score & $\begin{array}{l}22.2 \\
(17.8 \text { to } 27.3)\end{array}$ & $\begin{array}{l}37.1 \\
\text { (31.9 to } 43.0)\end{array}$ & $\begin{array}{l}38.0 \\
\text { (32.6 to } 43.7)\end{array}$ & $\begin{array}{l}41.5 \\
\text { (35.9 to } 47.1)\end{array}$ & $\begin{array}{l}51.0 \\
\text { (45.3 to } 56.7 \text { ) }\end{array}$ & $\begin{array}{l}45.2 \\
\text { (39.6 to } 50.9 \text { ) }\end{array}$ & $\begin{array}{l}25.3 \\
\text { (20.6 to } 30.6)\end{array}$ & $\begin{array}{l}26.3 \\
\text { (21.6 to } 31.5)\end{array}$ \\
\hline AVE & 0.66 & 0.75 & 0.59 & 0.32 & 0.31 & 0.46 & 0.54 & 0.32 \\
\hline M & 0.68 & 1.30 & 0.73 & 1.17 & 1.92 & 3.55 & 1.09 & 4.52 \\
\hline SD & 0.64 & 0.78 & 0.74 & 0.57 & 0.55 & 0.86 & 0.76 & 1.39 \\
\hline CR & 0.85 & 0.94 & 0.89 & 0.72 & 0.76 & 0.52 & 0.76 & 0.63 \\
\hline
\end{tabular}

${ }^{*} \mathrm{P}<0.05,{ }^{*} \mathrm{P}<0.01$.

$\%$, prevalence of mental health symptoms; AVE, variance average extracted; CR, composite reliability; M, mean.

poor eating habits as latent variables. The assessment of the measurement model showed an adequate fit to the data $\left(\chi^{2} / \mathrm{df}=1065.04(637), \mathrm{p}<0.001 ; \mathrm{TLI}=0.91\right.$, CFI $=0.92$, SRMR $=0.07$ and RMSEA $=0.05$; $95 \%$ CI 0.043 to 0.053 ). Considering these results, we proceeded to test the hypotheses.

\section{Structural model}

The hypothesised structural model (figure 2) demonstrated an acceptable fit to the data $\left(\chi^{2} / \mathrm{df}=1131.98\right.$ (648), $\mathrm{p}<0.001 ; \mathrm{TLI}=0.90$, CFI $=0.91$, SRMR $=0.07$ and RMSEA $=0.05$; $95 \%$ CI 0.054 to 0.06 ). According to our hypothesis, variables related to mental health symptoms were associated with each other, and between all dimensions of coping strategies, excluding symptoms of depression and maladaptive and adaptative coping strategies. Distress was positively related to symptoms of

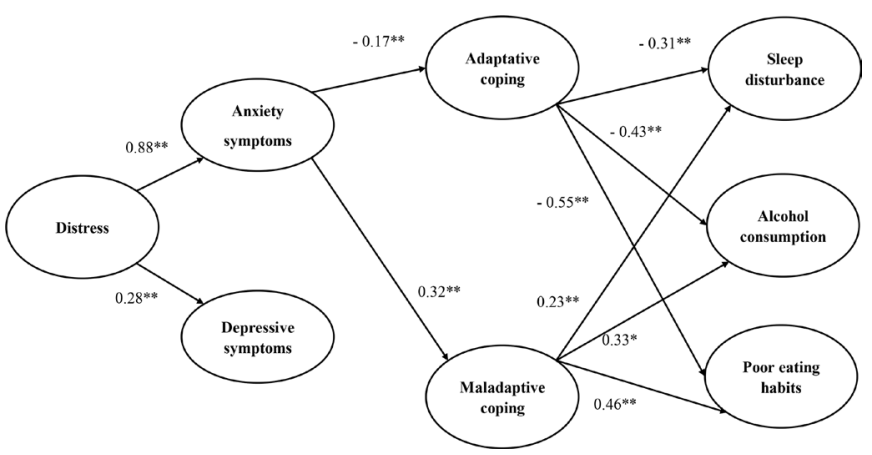

Figure 2 Hypothesised structural model for the

relationships between distress in eFootball players, anxiety/ depression symptoms, coping strategies and the outcome variables sleep disturbance, alcohol consumption and poor eating habits. *Standardised path coefficients significant at the 0.01 level; ${ }^{\star *}$ standardised path coefficients significant at the 0.05 level. Non-significant paths were excluded for visual simplicity. depression and anxiety. As expected, anxiety symptoms were positively associated with maladaptive coping strategies and negatively associated with adaptative coping strategies; that is, coping is a form of confrontation with anxiety symptoms. While adaptive coping strategies were negatively related to sleep disturbance, alcohol consumption and poor eating habits, a positive association between maladaptive coping strategies and the outcome variables was detected. The model proposed accounted for approximately $11 \%, 52 \%$ and $73 \%$ of sleep disturbance, alcohol consumption and poor eating habits, respectively.

\section{DISCUSSION}

The present study aimed to describe the prevalence of mental health symptoms and coping strategies and how they are associated in a satisfactory sample comprising eFootball players exclusively. According to our a priori hypothesis, distress was positively related to depression and anxiety symptoms, but only anxiety symptoms were linked with active coping strategies. Adaptative coping strategies were negatively related to sleep disturbance, alcohol consumption and poor eating habits. Conversely, maladaptive coping strategies were positively related to sleep disturbance, alcohol consumption and poor eating habits.

\section{Mental health symptoms}

Distress, depression symptoms, anxiety symptoms, sleep disturbance and poor eating habits were prevalent in eFootball players. Generally, athletes are vulnerable to mental health symptoms and disorders for factors such as stress related to their profession ${ }^{32}$ and the type of sports practice (ie, individual vs team sports), sports modality and various individual experiences (eg, emotional, mental, physical and social experiences, etc).${ }^{15}$ eFootball players may also face these factors in esports practice, 
though mental health symptoms and disorders are prevalent in professional athletes, being similar ${ }^{15}$ or even higher than the general population or former athletes. ${ }^{33}$ In the present study, the prevalence of anxiety and depression symptoms in eFootball players was similar to the prevalence reported in football players (anxiety in $38 \%$ and depression in $37 \%$ vs $25 \%-43 \%$, respectively). ${ }^{22}$ Still, when compared with the Portuguese population, ${ }^{34}$ eFootball players showed a higher prevalence of mental health disorders. Notably, Portugal is the second European country with the highest prevalence of mental health disorders $(23 \%)$, with anxiety being present in $17 \%$ and mood problems (ie, depression symptoms) in $8 \%$ of the population. ${ }^{34}$

\section{Association between distress and anxiety/depression symptoms}

In line with previous studies ${ }^{35-37}$ and according to our hypothesis, distress showed a positive association with both depression (hypothesis 1) and anxiety symptoms (hypothesis 2). A preliminary study on esports players concluded that different stressors pose significant challenges to the esports players; also, only those esports players who reported having higher levels of emotional control were able to reduce the intensity of the perceived stressor. ${ }^{35}{ }^{36}$ Hence, comparable to that happening in the traditional sports scene, the new opportunities to play at different professional levels can create more pressure, independently of esports participant's competitive experience. ${ }^{9}$ That means that understanding risk factors for distress in this unique population could be key to minimise its consequences. Similarly, when distress is already present, the way esports players cope with anxiety and depression symptoms might also have implications for their daily living.

\section{Depression symptoms and coping strategies}

Contrary to our previous hypothesis (hypothesis 3), no association was observed between depression symptoms and coping strategies. The results differ from previous studies that proposed a relationship between the general distress response, depression symptoms and coping strategies. ${ }^{37}{ }^{38}$ Different factors, such as a sample comprising mostly man and younger adults, could have hindered the results obtained since depression symptoms tend to be less prevalent in those populations. Further, comorbidity of depression symptoms and state anxiety may be part of a general 'psychological distress' response. ${ }^{37}$

\section{Anxiety symptoms and coping strategies}

Anxiety symptoms were associated with coping strategies (hypothesis 4), demonstrating a stronger association with maladaptive coping strategies than with adaptative ones. Individual predisposition to experience anxiety in a stressful situation and consequent coping styles could stimulate different responses to stressful conditions ${ }^{1537}$ in traditional sports and competitive esports athletes. ${ }^{10} 353940$ In line with Ntoumanis and Biddle's study, ${ }^{40}$ coping strategies could be influenced by how athletes perceived anxiety to be facilitative or debilitative of performance. As traditional sports, being part of the electronic sports competitive scene (ie, esports team, competitive calendar, etc) can also be perceived as challenging, threatening or harmful (eg, playing crucial competitions, coach-athlete conflicts, poor environment or internet conditions, and performance slumps), so appropriate coping skills are needed to deal effectively with situations that could increase anxiety symptoms, thus affecting, positively or negatively, esports performance.

\section{Coping strategies, sleep disturbance, alcohol consumption and poor eating habits}

Maladaptive coping strategies were positively associated with sleep disturbance, alcohol consumption and poor eating habits (hypothesis 5), while adaptive coping strategies showed a negative association (hypothesis 6). Two previous studies on esports players highlighted the link between coping mechanisms and mental illness. On a content analysis based on an interview with five highlevel competitive League of Legends players, emotional obstacles encountered by competitive gamers (eg, ineffective attentional control, limited ability to regulate emotions, trouble performing under pressure, etc) were related to the elements of optimal performance, such as inadequate coping strategies with anxiety symptoms. ${ }^{41}$ In another qualitative study with seven male esports players, the authors noted that despite the use of a range of different coping strategies, when in the presence of different stressors (eg, external scrutiny and criticism, audience, media interviews and problems with logistics), there was an overuse of maladaptive coping (ie, avoidance strategies). ${ }^{35}$ In the present study, we showed that sleep disturbance, alcohol consumption and eating habits were linked with the capacity to cope with anxiety symptoms and were present in response to the individual coping mechanisms.

\section{Coping strategies and sleep disturbance}

Based on our results, the presence of sleep disturbance resulting from maladaptive coping strategies due to anxiety symptoms might be a comorbid situation associated with sleep problems. ${ }^{15} 42$ The presence of sleep problems and disturbance due to inefficient anxiety control may impact esports performance and overall health. As such, sleep problems have been associated with athletic performance impairment across many sports. ${ }^{15}$ Different studies acknowledge the presence of sleep problems in esports players. ${ }^{43-45}$ Competition, which might induce distress and anxiety, as previously stated, has been associated with sleep disturbance before competition ${ }^{45}$ and prolonged sleep onset and offset times. ${ }^{45}$

\section{Coping strategies and alcohol consumption}

Poor mental health also seemed to be associated with alcohol consumption in athletes ${ }^{42}$ and the general Portuguese population. ${ }^{34}$ Factors such as the sport modality, 
competitive season, level of competition and extrinsic motivations for alcohol consumption (eg, experimentation, self-esteem improvement, alertness and energy improvement, team cohesion, etc) ${ }^{15}$ were associated with drinking problems in traditional sports. Still, they can also be a manifestation of inadequate maladaptive coping strategies. ${ }^{15}$

\section{Coping strategies and eating habits}

Adaptive coping (hypothesis 6) and maladaptive coping (hypothesis 5) were associated with different eating habits. Various studies suggested that psychological and emotional distress, including depression and anxiety, were associated with eating problems as an expression of maladaptive coping strategies. ${ }^{46}$ Consequently, as stated for the sleep disturbance and alcohol consumption, attention to predisposing factors to unhealthy eating habits is needed. Thus, ineffective coping strategies might occur due to specific stressors and anxiety. The strategies used by esports players in different situations might contribute to various factors that might hinder esports practice and contribute to other lifestyle problems that may disturb esports performance (eg, eating to suppress negative emotions and chronic stress). ${ }^{46}$

\section{Practical implications}

With the increasing number of esports players worldwide, we believe that health interventions focused on this unique population are needed to diagnose, manage properly and monitor mental health symptoms for esports players' general health and performance. Likewise, awareness and open communications could avoid unpleasant consequences of improper coping strategies. Ultimately, it is vital to understand how those problems impact esports performance.

From what is known about traditional sports athletes, ineffective coping mechanisms for common mental problems may have a negative impact on performance. However, given the association between distress, mental health symptoms and coping strategies, interventions aiming to empower esports players to cope with different stressors could impact psychological well-being and competitive esports performance. Various interventions have been proposed to improve adaptation to increased mental distress in traditional athletes and be considered for esports players. First, interventions to promote emotional stability could help lower perceived distress intensity, potentially improving performance and quality of life. ${ }^{10}$ Moreover, those strategies could have a long-term impact since psychological stress may impact behaviour and cognitive functioning. ${ }^{36}$ Second, sleep disturbance has been associated with poor competitive performance, and the adaptation of strategies aiming at an adequate balance between rest and recovery (eg, periodisation between practice and rest) might maximise adaptation to training and competition distress while reducing the likelihood of burnout or plateaus in performance in this population. ${ }^{48}$ Likewise, interventions at early signs of anxiety symptoms in esports players could be important to minimise harmful alcohol consumption, mostly by intervening on the eviction of alcohol use to reduce distress and negative emotion. Likewise, the availability of more healthy snacks instead of comfort/fast food during competitive periods of expected distress and anxiety could be a first step to promote healthy eating habits in people engaged with esports and reduce compulsive eating during stressed and anxious moments. ${ }^{46} 49$

\section{Limitations}

Limitations should be considered for this study, especially to enhance future studies on esports players. First, given the study design, causal relations and the findings' application to other contexts should be taken with caution. Here, we showed a theoretical model, based on Lazarus and Folkman's ${ }^{50}$ cognitive-relational theory of emotion and coping, about the association between mental health symptoms and coping strategies, so different models and paths could be studied Notably, it is important to state that the proposed model refers to variance average. Future studies, especially those with a longitudinal design, could be important to more robust interpretation of the results shown. Also, the study had a cross-sectional design, and generalisation and external validity might be compromised. Additionally, a convenience sample could predispose to selection bias, ${ }^{51}$ thus compromising generalisability. Moreover, with the present design, where we sent the survey to all registered efootball participants, despite being currently playing, there is no possibility to compare those who participated in the study with those who did not. Probably, those who had more interest in mental health were the ones who chose to fulfil the formulary. This fact might also contribute to a low response rate. For such, prevalence estimates of mental health problems in this sample could be relevant to understand this specific sample, given the novelty of the present study in esports research.

Therefore, future research on how distress within esports players impacts both health problems (eg, anxiety) and outcome behaviours (eg, alcohol consumption) through time could add important insights on short-term and long-term consequences of esports practice. Additionally, studies performed with a broader sample comprising esports players from different esports modalities (eg, Multiplayer Online Battle Arena, FirstPerson Shooter, etc), and comparisons between esports competition rank or professional status may add interesting insights on how to conduct further analysis and interventions for specific consequences of esports practice at higher levels. Given that different game types vary in their basic structural characteristics and gameplay, it is reasonable to assume that psychological symptoms related to different game types might also differ.

Second, we used self-reported questionnaires, and the external validity depends on the accuracy of the responses. It is known that questionnaire responses tend to underestimate problems (eg, depression) and behaviours (eg, 
alcohol consumption) with a negative connotation while overestimating positive ones. Additionally, the response rate was low, so close collaboration with the support staff of esports teams and team managers to promote participation in scientific investigations could be the first step to reach this population.

Third, there is no precise measurement tool to evaluate distress, specifically in esports settings. In the present study, the real cause of distress could not be defined. However, future studies should address the specific environmental constraints of the game to examine their impact. Additionally, we only classified coping strategies as adaptative or maladaptive. Still, we are aware that capturing other dimensions of coping would be appropriate because a dichotomic characterisation of coping may not adequately reflect the coping process's conceptual structure. Future research should include other forms of coping related to the esports scene.

\section{CONCLUSION}

This first-time study conducted in eFootball players demonstrated that mental health symptoms were present in eFootball players, especially for sleep disturbance and depression symptoms. Additionally, those symptoms were related. Distress was associated with both anxiety and depression symptoms. Moreover, adaptive coping strategies related to anxiety symptoms might be relevant to counteract the negative consequences on lifestyles habits, such as sleep disturbance, alcohol consumption and poor eating habits, in people engaged with esports. In our exploratory model, distress demonstrated a stronger association with anxiety symptoms. Conversely, anxiety symptoms were linked to coping strategies and showed a stronger association with maladaptive coping. Poor eating habit was the outcome variable more associated with active coping, followed by alcohol consumption. Thus, preventive measures on the distress pathway will be important to enhance health-related parameters in esports players.

\section{Author affiliations \\ ${ }^{1}$ Portugal Football School, Portuguese Football Federation, Oeiras, Portugal ${ }^{2}$ Research Center in Sports Sciences, Health Sciences and Human Development, University of Maia, ISMAI, CIDESD, Maia, Portugal \\ ${ }^{3} \mathrm{~N} 2 \mathrm{i}$, Polytechnic Institute of Maia, Maia, Porto, Portugal \\ ${ }^{4}$ CIPER, Interdisciplinary Center for the Study of Human Performance, University of Lisbon, Faculty of Human Kinetics, Lisbon, Portugal \\ ${ }^{5}$ Amsterdam Collaboration on Health \& Safety in Sports, Department of Public and Occupational Health, Amsterdam Movement Sciences, Amsterdam UMC, University Medical Centers - Vrije Universiteit Amsterdam, Amsterdam, Netherlands ${ }^{6}$ Section of Sports Medicine, University of Pretoria, Pretoria, South Africa ${ }^{7}$ Amsterdam UMC, University of Amsterdam, Department of Orthopaedic Surgery, Amsterdam Movement Sciences, Meibergdreef 9, Amsterdam, Netherlands ${ }^{8}$ Amsterdam Collaboration on Health \& Safety in Sports (ACHSS), Amsterdam UMC IOC Research Center of Excellence, Amsterdam, Netherlands \\ ${ }^{9}$ Research Center in Sports Sciences, Health Sciences and Human Development, CIDESD, Portugal \\ ${ }^{10}$ CIDEFES, Universidade Lusófona, Lisboa, Portugal}

Twitter Evert Verhagen @evertverhagen and Vincent Gouttebarge @VGouttebarge
Acknowledgements We acknowledge the contributions of Portuguese Football Association (FPF) Intelligence department and FPF Esports coordination team that aided the efforts of the authors for data collection.

Contributors All authors contributed to this original research. Conceptualisation, data curation and formal analysis: AMP and PT; investigation and visualisation: AMP and JB; methodology: AMP, PT and JB; project administration and resources: JB; software: PT; supervision: $\mathrm{EV}, \mathrm{PF}$ and JB; validation: AMP, JB, EV and VG; writing (original draft): AMP; writing-review and editing, AMP, PT, EV, VG, PF and JB.

Funding The authors have not declared a specific grant for this research from any funding agency in the public, commercial or not-for-profit sectors.

\section{Competing interests None declared.}

Patient and public involvement Patients and/or the public were not involved in the design, conduct, reporting or dissemination plans of this research.

\section{Patient consent for publication Not applicable.}

Ethics approval The procedures have been validated by the Portuguese Football Association (FPF) Data Protection Office and de FPF Esports department. The study was approved by the Portugal Football School Ethics Committee (CE PFS $\left.n^{\circ} 1 / 2021\right)$. An electronic informed and voluntary consent, following the Code of Ethics of the World Medical Association, containing the study's objectives was presented to all participants in the invitation to participate. The FPF Data Protection Office protected privacy rights.

Provenance and peer review Not commissioned; externally peer reviewed.

Data availability statement Data are available upon reasonable request. Data are available upon reasonable request at dpo@fpf.pt.

Open access This is an open access article distributed in accordance with the Creative Commons Attribution Non Commercial (CC BY-NC 4.0) license, which permits others to distribute, remix, adapt, build upon this work non-commercially, and license their derivative works on different terms, provided the original work is properly cited, appropriate credit is given, any changes made indicated, and the use is non-commercial. See: http://creativecommons.org/licenses/by-nc/4.0/.

\section{ORCID iDs}

Ana Monteiro Pereira http://orcid.org/0000-0002-0495-9230

Evert Verhagen http://orcid.org/0000-0001-9227-8234

Vincent Gouttebarge http://orcid.org/0000-0002-0126-4177

\section{REFERENCES}

1 NewZoo. 2020 games market report | April update. 2020.

2 NewZoo. Global Esports \& Live Streaming Market Report, 2021.

3 Baltezarević R, Baltezarević B. The impact of video games on the esports formation. FU Phys Ed Sport 2018;16:137.

4 Bányai F, Griffiths MD, Király O, et al. The psychology of Esports: a systematic literature review. J Gamb/ Stud 2019;35:351-65.

5 Jonasson K, Thiborg J. Electronic sport and its impact on future sport. Sport Soc 2010;13:287-99.

6 Adamus T. Playing Computer Games as Electronic Sport: In Search of a Theoretical Framework for a New Research Field. In: Computer games and new media cultures, 2012: 477-90.

7 García-Lanzo S, Chamarro A. Basic psychological needs, passion and motivations in amateur and semi-professional eSports players. Revista de Psicologia, Ciències de l'Eduació i de l'Esport 2018;36:10.

8 Pereira AM, Brito J, Figueiredo P, et al. Virtual sports deserve real sports medical attention. BMJ Open Sport Exerc Med 2019;5:e000606.

9 Cottrell C, McMillen N, Harris BS. Sport psychology in a virtual world: considerations for practitioners working in eSports. Journal of Sport Psychology in Action 2019;10:73-81.

10 Poulus D, Coulter TJ, Trotter MG, et al. Stress and coping in Esports and the influence of mental Toughness. Front Psychol 2020;11:628.

11 World Health Organization. Global health estimates. Geneva: World Health Organization, 2017

12 GBD 2019 Risk Factors Collaborators. Global burden of 87 risk factors in 204 countries and territories, 1990-2019: a systematic analysis for the global burden of disease study 2019. Lancet 2020;396:1223-49.

13 World Health Organization. Global status report on alcohol and health 2018 (License: CC BY-NC-SA 3.0 IGO. World Health Organization, 2018. https://apps.who.int/iris/handle/10665/274603

14 Laposky AD, Van Cauter E, Diez-Roux AV. Reducing health disparities: the role of sleep deficiency and sleep disorders. Sleep Med 2016;18:3-6. 
15 Reardon CL, Hainline B, Aron CM, et al. Mental health in elite athletes: international Olympic Committee consensus statement (2019). Br J Sports Med 2019;53:667-99.

16 van Hoffen MFA, Twisk JWR, Heymans MW, et al. Psychological distress screener for risk of future mental sickness absence in nonsicklisted employees. Eur J Public Health 2016;26:510-2.

17 Goldberg DP, Gater R, Sartorius N, et al. The validity of two versions of the GHQ in the who study of mental illness in general health care. Psychol Med 1997;27:191-7.

18 Carver CS. You want to measure coping but your protocol's too long: consider the brief cope. Int J Behav Med 1997;4:92-100.

19 Cella D, Riley W, Stone A, et al. The patient-reported outcomes measurement information system (PROMIS) developed and tested its first wave of adult self-reported health outcome item banks: 2005-2008. J Clin Epidemiol 2010;63:1179-94.

20 Bradley KA, DeBenedetti AF, Volk RJ, et al. AUDIT-C as a brief screen for alcohol misuse in primary care. Alcohol Clin Exp Res 2007;31:1208-17.

21 Van der Veer T, Frings-Dresen MHW, Sluiter JK. Health behaviors, care needs and attitudes towards self-prescription: a crosssectional survey among Dutch medical students. PLoS One 2011;6:e28038.

22 Gouttebarge V, Backx FJG, Aoki H, et al. Symptoms of common mental disorders in professional football (soccer) across five European countries. J Sports Sci Med 2015;14:7.

23 Braam C, van Oostrom SH, Terluin B, et al. Validation study of a distress screener. J Occup Rehabil 2009;19:231-7.

24 Pais Ribeiro J, Neto C, Silva M, et al. Further validation of the GOLDBERG 28 items general health questionnaire. Psic., Saúde \& Doenças 2015;16:278-85.

25 General Health Questionnaire. Gl assessment, 2020. Available: https://www.gl-assessment.co.uk/products/general-healthquestionnaire-ghq/ [Accessed $30 \mathrm{Jul}$ 2020].

26 Pais-Ribeiro J, Rodrigues A. Questões acerca do coping: a propósito do estudo de adaptação do brief cope. Psicologia, Saúde \& Doença 2004:5:12.

27 PROMIS. Health measures, 2020. Available: https://www. healthmeasures.net/explore-measurement-systems/promis [Accessed 30 Jul 2020].

28 Faul F, Erdfelder E, Buchner A, et al. Statistical power analyses using G*Power 3.1: tests for correlation and regression analyses. Behav Res Methods 2009;41:1149-60.

29 Hair JF, Black B, Babin B. Multivariate data analysis: a global perspective. 7th ed. Upper Saddle River NJ: Pearson Education, 2014.

30 Williams J, Mackinnon DP. Resampling and distribution of the product methods for testing indirect effects in complex models. Struct Equ Modeling 2008;15:23-51.

31 Nevitt J, Hancock G. Performance of bootstrapping approaches to model test statistics and parameter standard error estimation in structural equation modeling. Structural Equation Modeling: $A$ Multidisciplinary Journal 2001;8:353-77.

32 Souter G, Lewis R, Serrant L. Men, mental health and elite sport: a narrative review. Sports Med Open 2018;4:57.
33 Gouttebarge V, Castaldelli-Maia JM, Gorczynski P, et al. Occurrence of mental health symptoms and disorders in current and former elite athletes: a systematic review and meta-analysis. Br J Sports Med 2019;53:700-6.

34 Saúde CNde. Sem MAIS tempo a perder - Saúde mental em Portugal: um desafio para a próxima década. Lisboa: CNS, 2019.

35 Smith MJ, Birch PDJ, Bright D. Identifying stressors and coping strategies of elite Esports competitors. Int J Gaming Comput Mediat Simul 2019;11:22-39.

36 Cohen Jl. Stress and mental health: a biobehavioral perspective. Issues Ment Health Nurs 2000;21:185-202.

37 Endler NS, Parker JDA. State and trait anxiety, depression and coping styles. Aust J Psychol 1990;42:207-20.

$38 \mathrm{McNicol} \mathrm{ML}$, Thorsteinsson EB, Addiction I. Internet addiction, psychological distress, and coping responses among adolescents and adults. Cyberpsychol Behav Soc Netw 2017;20:296-304.

39 Raffety BD, Smith RE, Ptacek JT. Facilitating and debilitating trait anxiety, situational anxiety, and coping with an anticipated stressor: a process analysis. J Pers Soc Psychol 1997;72:892-906.

40 Ntoumanis N, Biddle SJH. Relationship of intensity and direction of competitive anxiety with coping strategies. Sport Psychol 2000;14:360-71.

41 Himmelstein D, Liu Y, Shapiro JL. An exploration of mental skills among competitive League of legend players. Int J Gaming Comput Mediat Simul 2017;9:1-21.

42 Kenney SR, Lac A, Labrie JW, et al. Mental health, sleep quality, drinking motives, and alcohol-related consequences: a path-analytic model. J Stud Alcohol Drugs 2013;74:841-51.

43 Rudolf K, Bickmann P, Froböse I, et al. Demographics and health behavior of video game and eSports players in Germany: the eSports study 2019. Int J Environ Res Public Health 2020;17:1870.

44 Lee S, Bonnar D, Roane B, et al. Sleep characteristics and mood of professional Esports athletes: a Multi-National study. Int J Environ Res Public Health 2021:18:664

45 Gomes MA, Narciso FV, de Mello MT, et al. Identifying electronicsport athletes' sleep-wake cycle characteristics. Chronobiol Int 2021;38:1-8.

46 Hemmingsson E. A new model of the role of psychological and emotional distress in promoting obesity: conceptual review with implications for treatment and prevention. Obes Rev 2014;15:769-79.

47 Fanelli Kuczmarski M, Cotugna N, Pohlig RT, et al. Snacking and diet quality are associated with the coping strategies used by a socioeconomically diverse urban cohort of African-American and white adults. J Acad Nutr Diet 2017:117:1355-65.

48 Baker J, Young B. 20 years later: deliberate practice and the development of expertise in sport. Int Rev Sport Exerc Psychol 2014;7:135-57.

49 Cemelli CM, Burris J, Woolf K. Video games impact lifestyle behaviors in adults. Top Clin Nutr 2016;31:96-110.

50 Lazarus RS, Folkman S. Transactional theory and research on emotions and coping. Eur J Pers 1987;1:141-69.

51 Delgado-Rodríguez M, Llorca J. Bias. J Epidemiol Community Health 2004;58:635-41. 